\title{
Stress versus immunity
}

\begin{abstract}
The aim of this mini-review is to discuss the effects of early life stress on some aspects of the immune response. Results from research models applied to study this subject, showed that stimuli that cause stress during early life alters the development of offspring's immune system, making it more prone to suffer different diseases.
\end{abstract}

Keywords: stress, prenatal stress, immune system
Volume 5 Issue 2 - 2018

\author{
Ana Cecilia Liaudat, Pablo Bosch, Nancy \\ Rodriguez \\ Department of Molecular Biology, National University of Río \\ Cuarto, Argentina
}

\begin{abstract}
Correspondence: Nancy Rodriguez, Department of Molecular Biology, Faculty of Exact, Physical-chemical and Natural Sciences, National University of Río Cuarto, National Route $36 \mathrm{Km601}$, X5804ZAB Rio Cuarto, Córdoba, Argentina, Tel 54-3584676232,Email nmarioli@exa.unrc.edu.ar
\end{abstract}

Received: September 18, 2017 | Published: April 13, 2018

\section{Introduction}

Stress can be defined as a real or supposed threat to physical or psychological integrity of an individual, resulting in a physiological and /or behavioral response. ${ }^{1}$ The degree of damage caused by the stress depends on the nature, intensity and duration of the stimuli as well as the stage of gestation at which the stressor is applied. ${ }^{2}$ Dhabhar $\&$ McEwen $^{3}$ showed that the activation of the physiologic stress response systems can also enhance immune function as evidenced by increase in allergic contact sensitivity or delayed-type hypersensitivity. In contrast, acute stress has no effect on the course of irritant contact sensitivity, an immune reaction that does not involve an Ag-specific memory response. Herbert \& Cohen ${ }^{4}$ suggested that objective stressful events leas to larger immune changes than subjective self-reports of stress and that interpersonal events are related to different immune outcomes than non-social events.

\section{Results}

Studies conducted in our laboratory demonstrated that adult rats prenatal stressed exposed to acute postnatal stress stimuli had fewer corticosterone levels than control animals. Postnatal stress decreased leucocyte and lymphocyte numbers while increased neutrophils; the effect was smaller in prenatally stressed animals. ${ }^{2}$ Furthermore, in a stress situation, prenatally stressed rats had lower $\mathrm{T}$ cell proliferation ${ }^{5}$ and IL-2 release. ${ }^{6}$

\section{Discussion}

When stress situations alter the endogenous environment of a pregnant mother, it exerts profound influences on the development of an organism and may predispose to adaptive disturbances in postnatal life. Chronic prenatal exposure to a severe stressor such as immobilization alters hypothalamic-pituitary-gonadal axis activity as shown by the increased maternal corticosterone concentration. ${ }^{2}$ The studies performed in our laboratory reflects that the application of stress stimuli during pregnancy in rats affects the hypothalamicpituitary-adrenal axis of male offspring's and the time to reach sexual maturity. It also produces weight loss, ${ }^{7}$ modifies the immunological cells distribution ${ }^{5}$ and produces alterations in adrenal gland physiology. ${ }^{8}$
There is a very close relationship between neuroendocrine and immune system, interaction mostly mediated by adrenal stress hormones. They are the major mediators of leucocyte redistribution. Prenatal stressed animals showed lower $\mathrm{T}$ cell proliferation and decreased IL-2 release ${ }^{6}$ and predisposed the offspring to an allergic episode. ${ }^{9}$ Student present in forty-two college showed that interleukins levels were also altered by stress situation as school examination. Psychologic stress causes increased levels of IL-1 $\beta$, IL6 , and IL-10 and decreased Interferon gamma production. ${ }^{10}$ Recently, Laukova $\mathrm{M}$ et al. ${ }^{11}$ demonstrating that repeated stress via alteration in catecholamines exaggerates the inflammatory response likely by recruiting peripheral monocytes and neutrophils to the spleen. Mayer et al. ${ }^{2}$ also showed redistribution in the total number of leucocytes, lymphocytes and neutrophils produce by chronic stress stimuli which could be related to the reduced ability of these cells to access immunological organs.

The remote and cumulative ancestral stress programs central metabolic pathways that are involved in catecholamine biosynthesis, immune responses, and microbial host interactions. ${ }^{12}$ Stress during early life increased the number of fecal boli and alters the fecal microbiota in response to a novel stress. ${ }^{13}$ During the first day of pregnancy, bacterial communities within maternal gut and vaginal compartments can impact the regulation of host immunity, metabolism, neurodevelopment, behavior and offspring development. ${ }^{14}$

The immune cells present in Central Nervous System were also affected by prenatal stress situation. Prenatal stress altered morphology and biological activity of microglia cells and contributed to the behavioral changes observed in depression in adulthood. ${ }^{15}$

\section{Conclusion}

It is important to denote that the relationship between stress and immune function is complex, but previous studies showed that repeated chronic stress stimuli during pregnancy can produced several alterations in offspring immune system which predispose to health disorders.

\section{Acknowledgements}

None. 


\section{Conflict of interest}

The authors report no conflicts of interest. The authors alone are responsible for the content and writing of this paper.

\section{References}

1. Vigas M. Contribution to the understanding of the stress concept. In: Usdin E, Kvetnansky R, Kopin IJ, editors. Catecholamines and stress: recent advance. New York: Elsevier; 1980:573.

2. Mayer N, Greco C, Bertuzzi M, et al. Immobilization stress responses in adult rats exposed in utero to immobilization. Stress Health. 2011;27(2):e1-10.

3. Dhabhar FS, McEwen BS. Stress-induced enhancement of antigenspecific cell-mediated immunity. J Immunol. 1996;156(7):2608-2615.

4. Herbert TB, Cohen S. Stress and immunity in humans: a meta-analytic review. Psychosom Med. 1993;55(4):364-379.

5. Liaudat AC, Rodríguez N, Vivas A, et al. Effect of early stimulation on some immune parameters in a model of prenatally stressed rats. International Journal of Psychological Studies. 2012;4:73-82.

6. Falcone EG, Liaudat AC, Alustiza FE, et al. IL-2 is involved in immune response of prenatally stressed rats exposed to postnatally stimulation. Austral Journal of Veterinary Sciences. 2017;49:113-118.

7. Rodríguez N, Mayer N, Gauna HF. Effects of prenatal stress on male offspring sexual maturity. Biocell. 2007;31(1):67-74.
8. Liaudat AC, Rodríguez N, Chen S, et al. Adrenal response in offspring male rats exposed to prenatal stress and to early postnatal stimulation. Biotech Histochem. 2015;90(6):432-438.

9. Moustaki M, Tsabouri S, Priftis K, et al. Prenatal stress enhances susceptibility to allergic diseases of offspring. Endocr Metab Immune Disord Drug Targets. 2017;17(4):255-263.

10. Paik IH, Toh KY, Lee C, et al. Psychological stress may induce increased humoral and decreased cellular immunity. Behav Med. 2000;26(3):139-141.

11. Laukova M, Vargovic P, Rokytova I, et al. Repeated stress exaggerates lipopolysaccharide-induced inflammatory response in the rat spleen. Cell Mol Neurobiol. 2017;38(1):195-208.

12. Kiss D, Ambeskovic M, Montina T, et al. Stress transgenerationally programs metabolic pathways linked to altered mental health. Cell Mol Life Sci. 2016;73(23):4547-4557.

13. O'Mahony SM, Marchesi JR, Scully P, et al. Early life stress alters behavior, immunity, and microbiota in rats: implications for irritable bowel syndrome and psychiatric illnesses. Biol Psychiatry. 2009;65(3):263-267.

14. Jašarević E, Howard CD, Misic AM, et al. Stress during pregnancy alters temporal and spatial dynamics of the maternal and offspring microbiome in a sex-specific manner. Sci Rep. 2017;7:44182.

15. Ślusarczyk J, Trojan E, Głombik K, et al. Prenatal stress is a vulnerability factor for altered morphology and biological activity of microglia cells. Front Cell Neurosci. 2015;9:82. 